\title{
Effective Model Study of the Weak Finite Temperature Phase Transition
}

\author{
F. Karsch ${ }^{a}$, T. Neuhaus ${ }^{a}$ and A. Patkós ${ }^{b}$ \\ a Fakultät für Physik, Universität Bielefeld, 33615 Bielefeld, Germany \\ b Institute of Physics, Eötvös University, H-1088, Puskin u. 5-7, Budapest, Hungary.
}

An effective 3-d 4-component scalar model is studied in order to determine the effects of order parameter fluctuations on the electroweak phase transition. Its lattice formulation contains also the effects of magnetic screening and is investigated both with mean field and Monte Carlo techniques. Numerical results point in the direction of a weakening of the first order phase transition at a value of the Higgs mass $m_{H}=35 \mathrm{GeV}$.

\section{INTRODUCTION}

The tiny matter-antimatter asymmetry observed on cosmic scales requires explanation in the framework of standard cosmology. Baryon number violating processes are known to occur in the electro-weak theory and are, in fact, expected to occur frequently at high temperatures. The occurence of a non-vanishing matter-antimatter asymmetry, however, is expected to be possible only, if the universe evolved through some nonequilibrium stages. If the electroweak phase transition was of first order (discontinuous) nature, than at temperatures $T_{c} \sim 100 \mathrm{GeV}$ there might have been a chance for developing the observed baryon asymmetry of the universe.

The very active present day investigation of this question is based on perturbative evaluation of the effective potential of the Higgs-field. Renewed interest in the field is due to the observation that the non-perturbatively generated screening masses for the gauge bosons as well as thermal fluctuations of the order parameter [1] might substantially modify the strength of the first order electroweak phase transition and eventually could lead to a second order transition, if the magnetic screening mass becomes too large [2]. Thus the perturbative calculations $[3,4]$ lead to constraints on the magnetic screening mass required for a first order transition and also lead to other predictions, like for instance the latent heat and surface tension. In this work we study the influence of the finite temperature fluctuations of the Higgs-field on these parameters. Specifically we will study an effective 3-d theory for the Higgsfield, which has been obtained from the $(3+1)$ dimensional SU(2)-Higgs model in two steps:

- Dimensional reduction: One integrates over all non-static Matsubara fields at one-loop level. Since these modes are massive, no infrared sensitivity is expected, their perturbative integration seems to be well-founded.

- Elimination of gauge degrees of freedom: The theory resulting from the first step is a 3-dimensional Gauge-Higgs model, where in addition, also an isovector field, the fourth component of the gauge fields, is present. In order to reduce the theory further one integrates over the magnetic gauge degrees of freedom and the isovector scalar. According to the improved perturbative treatments, the infrared stability can be ensured, if one includes into the result of the "naive" 3-dimensional 1-loop calculation the electric screening mass and an additional magnetic screening mass. Especially the second of them lacks, however, firm theoretical basis.

As a result of this approach one arrives at a three dimensional effective Lagrangian for the Higgs field alone

$$
\begin{gathered}
\mathcal{L}_{3 D}=\frac{1}{T}\left[\frac{1}{2} M_{\phi}^{2} \phi^{\dagger} \phi+\frac{\lambda}{24}\left(\phi^{\dagger} \phi\right)^{2}+\frac{1}{2} \partial_{i} \phi^{\dagger} \partial_{i} \phi\right. \\
\left.-\frac{T}{2 \pi}\left(M_{A}^{2}+\frac{1}{4} g^{2} \phi^{\dagger} \phi\right)^{3 / 2}-\frac{T}{4 \pi}\left(M_{\rho}^{2}+\frac{1}{4} g^{2} \phi^{\dagger} \phi\right)^{3 / 2}\right]
\end{gathered}
$$


where the nonperturbative magnetic screening mass is $M_{A}$ and the perturbative results for $M_{\phi}$ and $M_{\rho}$ are given by

$$
\begin{gathered}
M_{\phi}^{2}=m_{R}^{2}+\left(\frac{3}{16} g^{2}+\frac{1}{12} \lambda\right) T^{2}-C T \Sigma_{c} \\
M_{\rho}^{2}=\frac{5}{6} g^{2} T^{2} .
\end{gathered}
$$

$\Sigma_{c}$ is the linearly divergent integral $\int_{p} 1 / p^{2}, C=$ $\lambda$ in perturbation theory. The above parameters $m_{R}$ and $\lambda$ are close but not identical to the temperature zero values of the Higgs-mass and the quartic coupling of the $D=4$ theory. They pick up a renormalization correction, which can be worked out when temperature independent terms of the effective potential in our approach are compared with the zero temperature effective potential of the full theory. These contributions are however small at the value of the Higgs-mass, which is considered in this paper. We note that the effective $3 D$ action [5] reproduces the selfconsistent gap equations for the screening masses widely used in the perturbative treatment of the phase transitions $[3,4]$. We thus expect that an analysis of the above effective $3 D$ model with nonperturbative methods will yield valuable information for the influence of screening masses on the nature of the electroweak phase transition.

\section{THE LATTICE ACTION}

We consider the following lattice action

$$
\begin{gathered}
\mathcal{S}_{3 D, \text { Latt }}=\sum_{x}\left[\frac{1}{2} \frac{1}{\kappa} \phi_{x}^{\dagger} \phi_{x}+\frac{\Theta \lambda}{24}\left(\phi_{x}^{\dagger} \phi_{x}\right)^{2}\right. \\
-\frac{1}{2} \sum_{\nu}\left(\phi_{x}^{\dagger} \phi_{x+\nu}+\phi_{x}^{\dagger} \phi_{x-\nu}\right) \\
\left.-\frac{g^{3} \Theta^{3 / 2}}{16 \pi}\left\{\left(\gamma_{A} \Theta+\phi_{x}^{\dagger} \phi_{x}\right)^{3 / 2}+\frac{1}{2}\left(\gamma_{\rho} \Theta+\phi_{x}^{\dagger} \phi_{x}\right)^{3 / 2}\right\}\right] .
\end{gathered}
$$

Hereby denotes $\Theta=a T$ the physical temperature in units of $a, \kappa$ is the hopping parameter which is related to the temperature dependent Higgs mass by $\kappa=\left(M_{\Phi}^{2} a^{2}+6\right)^{-1}$. The couplings $g^{2}$ and $\lambda$ denote the corresponding zero temperature gauge field and quartic couplings. We fix the value of $g^{2}$ by choosing a W-mass $m_{W}=80 \mathrm{GeV}$ and the vacuum expectation value of the Higgs-field to be $v_{0}=246 \mathrm{GeV}$. In addition $\gamma_{A}$ and $\gamma_{\rho}$ are dimensionless constants appearing in the definitions of the electric and magnetic screening masses $\left(M_{\boldsymbol{A}, \rho}^{2}=\gamma_{\boldsymbol{A}, \rho} g^{2} T^{2}\right)$ respectively. In resummed perturbation theory $\gamma_{\rho}=\frac{10}{3}$ and $\gamma_{A} \propto O\left(g^{2}\right)$. Using for $\gamma_{\rho}$ its perturbative value we choose for the parameter $\gamma_{A}$ a value of $\gamma_{A}=\gamma_{B} \frac{4}{9 \pi^{2}}$ and keep $\gamma_{B}$ as a free parameter. We then consider a value for the Higgs-mass $m_{H}=35 \mathrm{GeV}$, which determines the quartic quartic coupling $\lambda=3 m_{H}^{2} / v_{0}^{2}$. This choice is motivated by a recent work of Kajantie et al.[6] in order to facilitate a comparison of their results with our approach. After fixing all these parameters we are left with the two free parameters $\Theta$ and $\kappa$. In the $\kappa-\Theta$ plane of couplings we expect a phase transition from a symmetric to a broken phase in the model. It means tuning $\kappa$ and $\Theta$ on the PT line approaching the Gaussian Fixed Point at $\Theta=0$ will allow the construction of the continuum limit.

\section{MEAN FIELD ANALYSIS}

The mean field calculation of $\mathcal{S}_{3 D, L a t t}$ exhibits the properties of the model in a qualitative way. In the $\kappa-\Theta$ plane of couplings we obtain scaling laws, e.g. for the order parameter jump $\Phi_{\text {latt }}$, the interface tension $\sigma_{l a t t}$ and the shape of the phase transition line $\kappa_{c}(\Theta)$

$$
\begin{gathered}
\Phi_{l a t t}=\frac{\Phi}{T_{c}} \Theta^{0.5} \\
\sigma_{l a t t}=\frac{\sigma}{T_{c}^{3}} \Theta^{2} \\
\left(\kappa_{c}(\Theta)^{-1}-6\right) \Theta^{2}=2 Z_{M F}
\end{gathered}
$$

with $Z_{M F}$ determined by the mean field equations. Fig. 1) shows the quantity $\sigma / T_{c}^{3}$ as a function of the magnetic screening mass parameter $\gamma_{B}$ and the Higgs-mass. It can be seen that for $\gamma_{B}=1$ the phase transition is first order up to values of the Higgs mass of about $85 \mathrm{GeV}$, turning then into a second order phase transition. These results are consistent with results of Buchmüller et al [3]. 


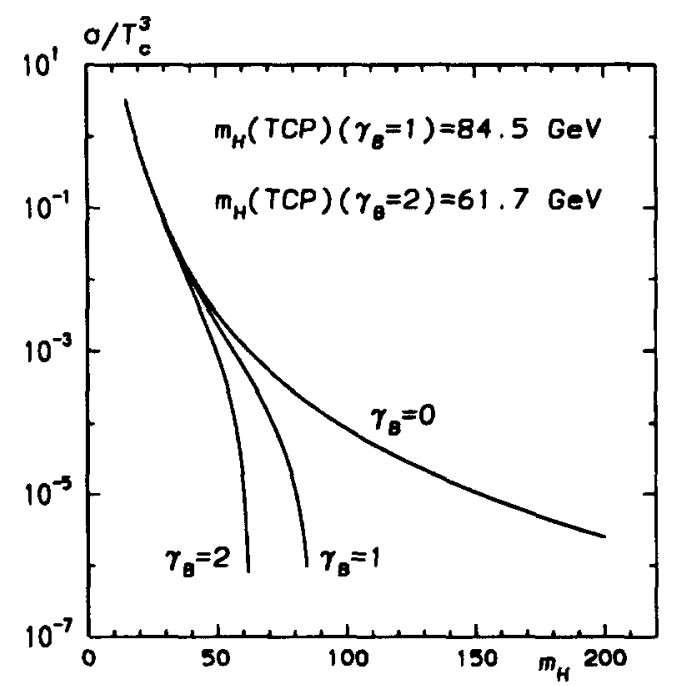

Figure 1. Mean field results for $\sigma / T_{c}^{3}$ as function of $\gamma_{B}$ and $m_{H}$. TCP denotes the end point of the first order regime.

\section{MONTE CARLO ANALYSIS}

In our present numerical evaluation we simulate $\mathcal{S}_{3 D, \text { Latt }}$ using a Multicanonical algorithm. We briefly summarize the numerical results of our simulation obtained so far in Table 1). The Table contains values for the jump of the order parameter and estimates for the interface tension as determined at $m_{H}=35$ and $\gamma_{B}=1$, as well as an estimate of the critical temperature, which is determined from the shape of the phase transition line. The interface tension estimates are obtained from ratios of probability distribution functions of certain operators in the metastable region of the model [7]. For a detailed description of the employed methods we refer to a forthcoming paper [8]. These results indicate a weakening of the $\mathrm{PT}$ as compared to the mean field calculations, e.g. see the comparison of interface tension estimators as compared to the mean field value. This result is quite interesting. It has to be contrasted with a recent claim by Kajantie et al.[6], which states that they see a somewhat stronger first order phase transition, than indicated by a 1-loop perturbative analysis of their model. They would attribute this effect to nonperturbative contributions from gauge field dynamics, while we would expect a weakening of the phase transition induced by fluctuations of the Higgs field. Thus a possible explanation could be that the full dynamics of the gauge fields operates in the direction of a strengthening of the first order phase transition.

Table 1

Numerical results (MC) in comparison to mean field analysis (MF). $L$ denotes the linear extent of the lattice. The interface free energy estimators from the Monte Carlo simulation use Binder's method.

\begin{tabular}{||c|c|c|c|c||}
\hline & $\mathrm{MF}$ & $\mathrm{MC}$ & $\Theta$ & $L$ \\
\hline $\bar{T}_{c}$ & 99.62 & 115 & - & - \\
\hline$\Phi / T_{c}$ & 1.32 & 1.10 & 5.0 & 16 \\
& - & 0.90 & 3.0 & 18 \\
\hline$\sigma / T_{c}^{3} 10^{2}$ & 2.42 & 0.15 & 5.0 & 8 \\
& - & 0.27 & 5.0 & 12 \\
& - & 0.38 & 5.0 & 16 \\
& - & 0.05 & 3.0 & 18 \\
\hline
\end{tabular}

\section{REFERENCES}

1. M. Gleiser and E. W. Kolb, Phys. Rev. D48 (1993)1560.

2. M. Dine, R. G. Leigh, P. Huet, A. Linde and D. Linde, Phys. Rev D46 (1992) 550.

3. W. Buchmüller, Z. Fodor, T. Helbig and D. Walliser, The weak electroweak phase transition, DESY 93-021, February 1993.

4. J. K. Espinosa, M. Quiros and F. Zwirner, Phys. Lett. B314 (1993) 206

5. A. Jakovác and A. Patkós, Z. f. Physik C60 (1993) 621

6. K. Kajantie, K. Rummukainen and M. Shaposhnikov, Nucl. Phys. B407 (1993) 356; K. Farakos, K. Kajantie, K. Rummukainen and M. Shaposhnikov, CERN TH 6973/93.

7. K. Binder, Z. Phys. B43 (1981) 119.

8. F. Karsch, T. Neuhaus and A. Patkós, in preparation. 